\title{
Brain and CSF Volumes in Fetuses and Neonates with Antenatal Diagnosis of Critical Congenital Heart Disease: A Longitudinal MRI Study
}

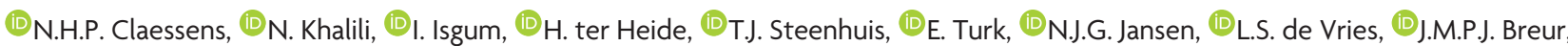 \\ (1) R. de Heus, and ${ }^{\circ}$ M.J.N.L. Benders
}

\begin{abstract}
BACKGROUND AND PURPOSE: Fetuses and neonates with critical congenital heart disease are at risk of delayed brain development and neurodevelopmental impairments. Our aim was to investigate the association between fetal and neonatal brain volumes and neonatal brain injury in a longitudinally scanned cohort with an antenatal diagnosis of critical congenital heart disease and to relate fetal and neonatal brain volumes to postmenstrual age and type of congenital heart disease.
\end{abstract}

MATERIALS AND METHODS: This was a prospective, longitudinal study including 61 neonates with critical congenital heart disease undergoing surgery with cardiopulmonary bypass $<30$ days after birth and MR imaging of the brain; antenatally ( 33 weeks postmenstrual age), neonatal preoperatively (first week), and postoperatively (7 days postoperatively). Twenty-six had 3 MR imaging scans; 61 had at least $1 \mathrm{fetal}$ and/or neonatal MR imaging scan. Volumes (cubic centimeters) were calculated for total brain volume, unmyelinated white matter, cortical gray matter, cerebellum, extracerebral CSF, and ventricular CSF. MR images were reviewed for ischemic brain injury.

RESULTS: Total fetal brain volume, cortical gray matter, and unmyelinated white matter positively correlated with preoperative neonatal total brain volume, cortical gray matter, and unmyelinated white matter $(r=0.5-0.58)$; fetal ventricular CSF and extracerebral CSF correlated with neonatal ventricular CSF and extracerebral CSF ( $r=0.64$ and 0.82). Fetal cortical gray matter, unmyelinated white matter, and the cerebellum were negatively correlated with neonatal ischemic injury ( $r=-0.46$ to -0.41 ); fetal extracerebral CSF and ventricular CSF were positively correlated with neonatal ischemic injury $(r=0.40$ and 0.23 ). Unmyelinated white matter:total brain volume ratio decreased with increasing postmenstrual age, with a parallel increase of cortical gray matter:total brain volume and cerebellum:total brain volume. Fetal ventricular CSF:intracranial volume and extracerebral CSF:intracranial volume ratios decreased with increasing postmenstrual age; however, neonatal ventricular CSF:intracranial volume and extracerebral CSF:intracranial volume ratios increased with postmenstrual age.

CONCLUSIONS: This study reveals that fetal brain volumes relate to neonatal brain volumes in critical congenital heart disease, with a negative correlation between fetal brain volumes and neonatal ischemic injury. Fetal brain imaging has the potential to provide early neurologic biomarkers.

ABBREVIATIONS: $C G M=$ cortical gray matter; $C H D=$ congenital heart disease; $E C S F=$ extracerebral CSF; ICV = intracranial volume; PMA = postmenstrual age; $\mathrm{TBV}=$ total brain volume; $\mathrm{UWM}=$ unmyelinated white matter; $\mathrm{VCSF}=$ ventricular $\mathrm{CSF} ; \mathrm{WMI}=$ white matter injury

$\mathbf{N}$ eurologic comorbidities are prevalent in neonates undergoing open heart surgery with the use of cardiopulmonary bypass for critical congenital heart disease (CHD) in the first weeks of life. ${ }^{1}$ Ischemic brain injury is common before and after the surgical procedure and is associated with neurodevelopmental disabilities throughout school age. ${ }^{2,3}$ In addition, delayed brain

Received November 11, 2018; accepted after revision February 27, 2019.

From the Departments of Neonatology (N.H.P.C., E.T., L.S.d.V., M.J.N.L.B.), Pediatric Cardiology (N.H.P.C., H.t.H., T.J.S., J.M.P.J.B.), Pediatric Intensive Care (N.H.P.C., N.J.G.J.), and Obstetrics (R.d.H.), Wilhelmina Children's Hospital, Utrecht, the Netherlands; Image Sciences Institute (N.K., I.I.), University Medical Center Utrecht, Utrecht, the Netherlands; and Department of Pediatrics (N.J.G.J.), Beatrix Children's Hospital, University Medical Center Groningen, Groningen, the Netherlands. Roel de Heus and Manon J.N.L. Benders share last authorship. growth with suggested antenatal onset has been reported in those with critical CHD. ${ }^{4,5}$

Antenatal studies have shown fetuses with critical CHD to demonstrate progressively reduced brain growth with enlarged CSF spaces compared with healthy fetuses. ${ }^{6}$ Smaller brain volumes in fetuses with critical CHD are thought to be the result of reduced antenatal cerebral oxygen delivery and cerebral oxygen

Please address correspondence to Nathalie Claessens, MD, PhD, Department of Neonatology, University Medical Centre Utrecht, KE 04.123.1, PO Box 85090, 3508 AB Utrecht, the Netherlands; e-mail: n.h.p.claessens-2@umcutrecht.nl; @UMCutrecht; @hetWKZ

三 Indicates article with supplemental on-line appendix and table.

Indicates article with supplemental on-line photo

http://dx.doi.org/10.3174/ajnr.A6021

AJNR Am J Neuroradiol 40:885-91 May 2019 www.ajnr.org 

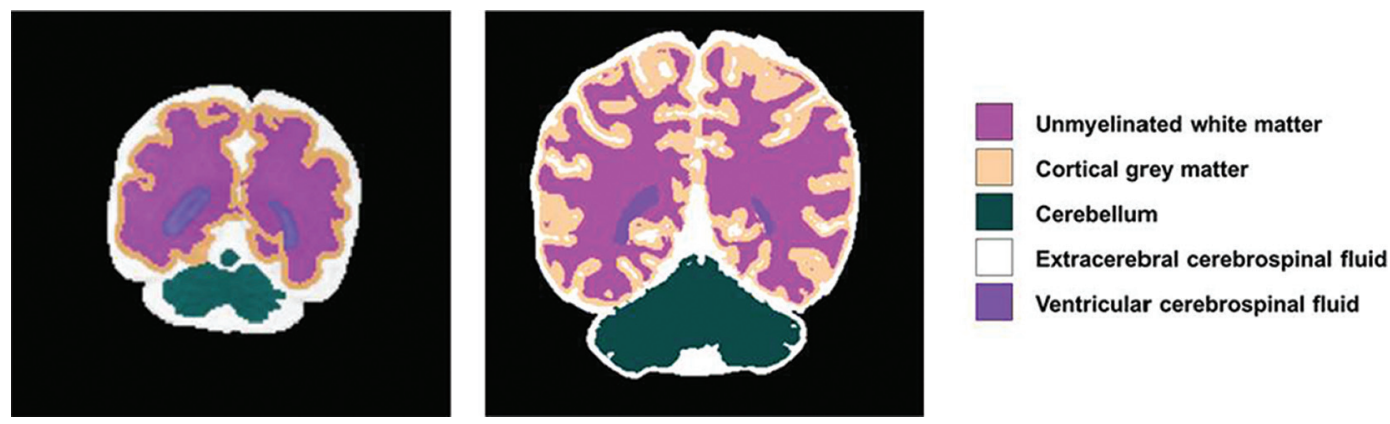

FIG 1. Fetal and neonatal brain tissue segmentation. Coronal T2-weighted segmented fetal and neonatal image of the same individual with transposition of the great arteries at 30 weeks of gestation and before the operation.

consumption, a consequence of the anatomic changes from the cardiac defect. ${ }^{5,7,8}$ The extent to which delayed fetal brain growth is transient or persists into the neonatal period remains undescribed. Revealing the association between fetal and neonatal delayed brain growth and acquired neonatal brain injury provides important information for the potential use of fetal brain imaging parameters as biomarkers for neurologic outcome in infants with critical CHD. This would also allow future implementation of interventions for improving brain growth before birth.

Using a prospective, longitudinal design, we investigated the correlation between fetal and neonatal preoperative and postoperative brain and CSF volumes, and the correlation of fetal brain and CSF volumes with neonatal ischemic brain injury. Our secondary aim was to examine trajectories of brain and CSF volumes in relation to postmenstrual age (PMA) during the third trimester of gestation and first weeks after birth in different cardiac defects.

\section{MATERIALS AND METHODS}

This was a prospective, longitudinal observational cohort study.

\section{Study Population}

Between May 2016 and December 2017, fetuses with an antenatal diagnosis of critical CHD requiring open heart surgery with the use of cardiopulmonary bypass at $<30$ days of life and referred to the Wilhelmina Children's Hospital Utrecht, a tertiary level hospital, underwent longitudinal MR imaging of the brain at 3 time points, antenatally (around 33 weeks PMA), neonatal preoperatively (within the first week of life), and neonatal postoperatively (around 7 days after the operation; range, 5-10 days). For this study, we excluded individuals with a genetic syndrome confirmed by antenatal karyotype or microarray (such as trisomy 21 or 22q11 deletion) or cerebral congenital malformations (such as corpus callosum agenesis). The local medical ethics board approved the study, and parental informed consent for the use of clinical data for research purposes was obtained.

\section{MR Imaging Acquisition}

All fetuses and neonates were scanned on a 3T MR imaging system (Achieva, Philips Healthcare, Best, the Netherlands). Fetal and neonatal MR images were reviewed by 2 independent researchers for the presence of congenital anomalies, parenchymal hemorrhage, ischemic brain injury, and ventriculomegaly (atrial diameter, $>10$ $\mathrm{mm}) .{ }^{9,10}$ Details are provided in the On-line Appendix.

\section{Quantitative 3D Volumetric Analysis}

Fetal and neonatal coronal T2-weighted images were automatically segmented into different tissue classes: extracerebral CSF (ECSF), ventricular CSF (VCSF), unmyelinated white matter (UWM), cortical gray matter (CGM), cerebellum, basal ganglia and thalamus, brain stem, and myelinated white matter (Fig 1), using a further adjusted approach for neonatal MR images. ${ }^{11}$ Volumes were calculated for all 8 tissue classes. All segmentations were manually checked for quality; no manual adjustments to the segmentations were made. Because basal ganglia, brain stem, and myelinated white matter were difficult to distinguish reliably on fetal T2-weighted images, these tissues were not taken into account for this study. Intracranial volume (ICV) was calculated as the sum of all 8 tissues classes; total brain volume (TBV), as the sum of UWM, CGM, cerebellum, basal ganglia and thalamus, brain stem, and myelinated white matter.

\section{Statistical Analysis}

For statistical analysis, R statistical and computing software, Version 3.5.0 (http://www.r-project.org/) was used. Clinical variables were predominantly not normally distributed; therefore, nonparametric tests were performed and medians (25th/75th centiles) were presented for continuous data. Counts (percentage) were presented for categoric data. To test the differences in baseline characteristics between individuals with and without fetal MR imaging, we used Mann-Whitney $U$ and Fisher exact tests. Univariable linear and nonlinear quadratic regression analysis was performed to examine the association between fetal brain and CSF volumes and neonatal (preoperative and postoperative) brain, and between CSF volumes and acquired neonatal brain injury, with correction for PMA at the time of scanning. Correlation coefficients with $95 \%$ confidence intervals were presented. Mixed-model analysis (R, nlme package 3.1-137) was performed to test the difference in brain and CSF volumes between different types of CHDs (fixed effect), including PMA (fixed effect), with the brain or CSF volume as dependent factors and individual subjects as random factors. A $P$ value $<.007$ was considered significant with correction for multiple comparisons.

\section{RESULTS}

\section{Study Population}

In the study period, 71 neonates with an antenatal diagnosis of critical CHD were born, of whom 8 died without any MR imaging of the brain. Sixty-three neonates with an antenatal diagnosis of 
Table 1: Baseline and MRI characteristics of the study population ${ }^{\mathrm{a}}$ Characteristics

\begin{tabular}{|c|c|}
\hline \multicolumn{2}{|c|}{ Maternal characteristics ( $n=29$; fetal MRI) } \\
\hline Maternal age (yr) & $30.5(27.8 / 35.3)$ \\
\hline Maternal body mass index & $22.8(20.8 / 23.9)$ \\
\hline Maternal smoking & $3(12 \%)$ \\
\hline Placental weight (g) & $455(375 / 510)$ \\
\hline \multicolumn{2}{|l|}{$\begin{array}{l}\text { Patient characteristics ( } n=61 \text {; total } \\
\text { study population) }\end{array}$} \\
\hline Female & $18(30)$ \\
\hline \multicolumn{2}{|l|}{ CHD groups } \\
\hline SVP & $21(34)$ \\
\hline TGA & $18(30)$ \\
\hline LVOTO & $22(36)$ \\
\hline Antenatal CHD diagnosis & $61(100)$ \\
\hline Twin & $1(2)$ \\
\hline Genetic disorder & $0(0)$ \\
\hline Additional congenital anomaly & $3(5)$ \\
\hline Gestational age (wk) & $39.1(38.4 / 40.1)$ \\
\hline Birth weight (g) & $3320(2970 / 3670)$ \\
\hline Birth weight (z score) & $-0.4(-0.8 / 0.3)$ \\
\hline Cesarean delivery & $18(31)$ \\
\hline Neonatal death & $6(10)$ \\
\hline \multicolumn{2}{|l|}{ MRI characteristics } \\
\hline Fetal, PMA (wks) & $33.4(32.7 / 34.1)$ \\
\hline Preoperative, days after birth & $5(3 / 6)$ \\
\hline Preoperative, PMA (wks) & $40.1(39.1 / 41.0)$ \\
\hline Postoperative, days after surgery & $8(7 / 9)$ \\
\hline Postoperative, PMA (wks) & $42.0(40.6 / 43.0)$ \\
\hline
\end{tabular}

Note:- LVOTO indicates left ventricle outflow tract obstruction; SVP, single ventricle physiology; TGA, transposition of the great arteries.

a Median and interquartile range or number with percentage are shown. No significant differences were seen between the primary and secondary study populations.

critical CHD had cardiac surgery within 30 days after birth, of whom, 2 were excluded because of a genetic syndrome, leaving 61 neonates eligible for this study. Thirty-one neonates underwent all 3 MR imaging scans; however, 5 had poor fetal imaging quality, leaving 26 with all $3 \mathrm{MR}$ imaging scans for the primary study aim. All 61 neonates were included for the secondary study aim: 26 plus an additional 21 with $2 \mathrm{MR}$ imaging scans and 14 with $1 \mathrm{MR}$ imaging scan (flowchart in On-line Figure). Baseline characteristics are presented in Table 1.

\section{Fetal and Neonatal MR Imaging: Conventional Analysis}

Two fetuses showed unilateral ventriculomegaly (atrial diameter, 10-15 mm). None of the fetuses showed parenchymal hemorrhage or ischemic brain injury.

At preoperative neonatal MR imaging, focal infarction was seen in $2(8 \%)$ and WM injury (WMI) in $2(8 \%)$. Punctate cerebellar hemorrhage was seen in $1(4 \%)$. Cumulatively (at preoperative and postoperative MR imaging), focal infarction was present in $7(28 \%)$ and WMI in 9 (38\%). Hypoxic-ischemic watershed injury was not seen in any neonates.

\section{Fetal Brain and CSF Volumes: Clinical Factors}

Fetal UWM was positively associated with fetal CGM $(r=0.87)$; fetal UWM, with fetal cerebellum $(r=0.60)$; and fetal CGM, with fetal cerebellum $(r=0.56)$. Fetal UWM:TBV (proportion of TBV occupied by UWM) was negatively associated with fetal CGM: TBV $(r=-0.51)$. Fetal VCSF and ECSF were negatively associated with fetal UWM:TBV ( $r=-0.39$ and -0.53 , respectively).

Fetal brain and CSF volumes (corrected for PMA at scanning)
Table 2: Association between fetal and neonatal MRI ${ }^{\mathrm{a}}$

\begin{tabular}{|c|c|c|c|}
\hline \multirow[b]{2}{*}{$\begin{array}{c}\text { Fetal } \\
\text { Volume }\end{array}$} & \multicolumn{3}{|c|}{ Neonatal } \\
\hline & $\begin{array}{c}\text { Preoperative } \\
\text { Volume }\end{array}$ & $\begin{array}{l}\text { Postoperative } \\
\text { Volume }\end{array}$ & $\begin{array}{l}\text { Ischemic } \\
\text { Brain Injury }\end{array}$ \\
\hline $\mathrm{TBV}^{\mathrm{b}}$ & & 0 & 0 \\
\hline$U^{b} M^{b}$ & & & $\square$ \\
\hline $\mathrm{CGM}^{\mathrm{b}}$ & 0 & 口 & $\square$ \\
\hline $\mathrm{CB}^{\mathrm{b}}$ & - & & $\square$ \\
\hline UWM:TBV & & 0 & $\overline{\mathbf{a}}$ \\
\hline CGM:TBV & 口 & & \\
\hline CB:TBV ${ }^{c}$ & & 0 & $\square$ \\
\hline$T B V: I^{C} V^{c}$ & & 口 & \\
\hline $\mathrm{VCSF}^{\mathrm{C}}$ & & & \\
\hline $\mathrm{ECSF}^{\mathrm{C}}$ & a & 0 & $\square$ \\
\hline VCSF:ICV & $\square$ & 0 & \\
\hline ECSF:ICV & $\overline{5}$ & - & \\
\hline
\end{tabular}

Note:-CB indicates cerebellum.

a Strength of correlation of fetal and neonatal preoperative volumes (first column); fetal and neonatal postoperative volumes (second column); fetal volumes and neonatal ischemic brain injury (third column). Square indicates moderate correlation (closed square, $r=0.3-0.5$; open square $r=-0.5$ to -0.3 ). Circle indicates strong correlation (closed circle, $r=0.5-0.8$; open circle, $r=-0.8$ to -0.5 ).

${ }^{\mathrm{b}}$ Result of linear regression analysis with correction for PMA at scanning.

c Result of quadratic regression analysis. Correlation coefficient and $95 \% \mathrm{Cl}$ are in the On-line Table.

were not associated with maternal body mass index, maternal age, maternal smoking, placental weight, or birth weight $z$ score (all $P$ values $>.007$ with Bonferroni correction for multiple comparisons). None of the mothers of the primary study population had preeclampsia or gestational diabetes.

\section{Brain and CSF Volumes: Correlation between Fetal and Neonatal MR Imaging}

Fetal volumes of CGM, UWM, and TBV correlated with preoperative neonatal volumes of CGM, UWM, and TBV, respectively (Table 2). Postoperatively, the association of fetal CGM and TBV with neonatal CGM and TBV, respectively, was still seen. Fetal VCSF, ECSF, VCSF: ICV, and ECSF:ICV correlated with preoperative neonatal VCSF, ECSF, VCSF:ICV, and ECSF:ICV, respectively. Postoperatively, the associations between fetal and neonatal MR imaging were still seen for ECSF and VCSF: ICV (Table 2 and On-line Table).

\section{Fetal Brain and CSF Volumes: Correlation with Neonatal Ischemic Brain Injury}

Baseline characteristics were not different between neonates with and without ischemic brain injury (data not shown). Both fetuses with antenatal ventriculomegaly had moderate-severe WMI before the operation $(100 \%)$.

The fetal TBV, CGM, UWM, and cerebellum were negatively associated with the presence of neonatal (either preoperatively or postoperatively acquired) ischemic brain injury (Table 2), in which fetal ECSF was positively associated with neonatal ischemic brain injury. The rate of preoperative ischemic brain injury was low and consequently was not examined separately in relation to fetal brain and CSF volumes.

\section{Fetal and Neonatal MR Imaging: Brain Volumes, PMA, and Type of CHD}

For the secondary study aim, the total study population of 61 subjects was analyzed. ICV, TBV, UWM, CGM, and the cerebellum all showed a linear association with PMA (Fig 2). The pro- 

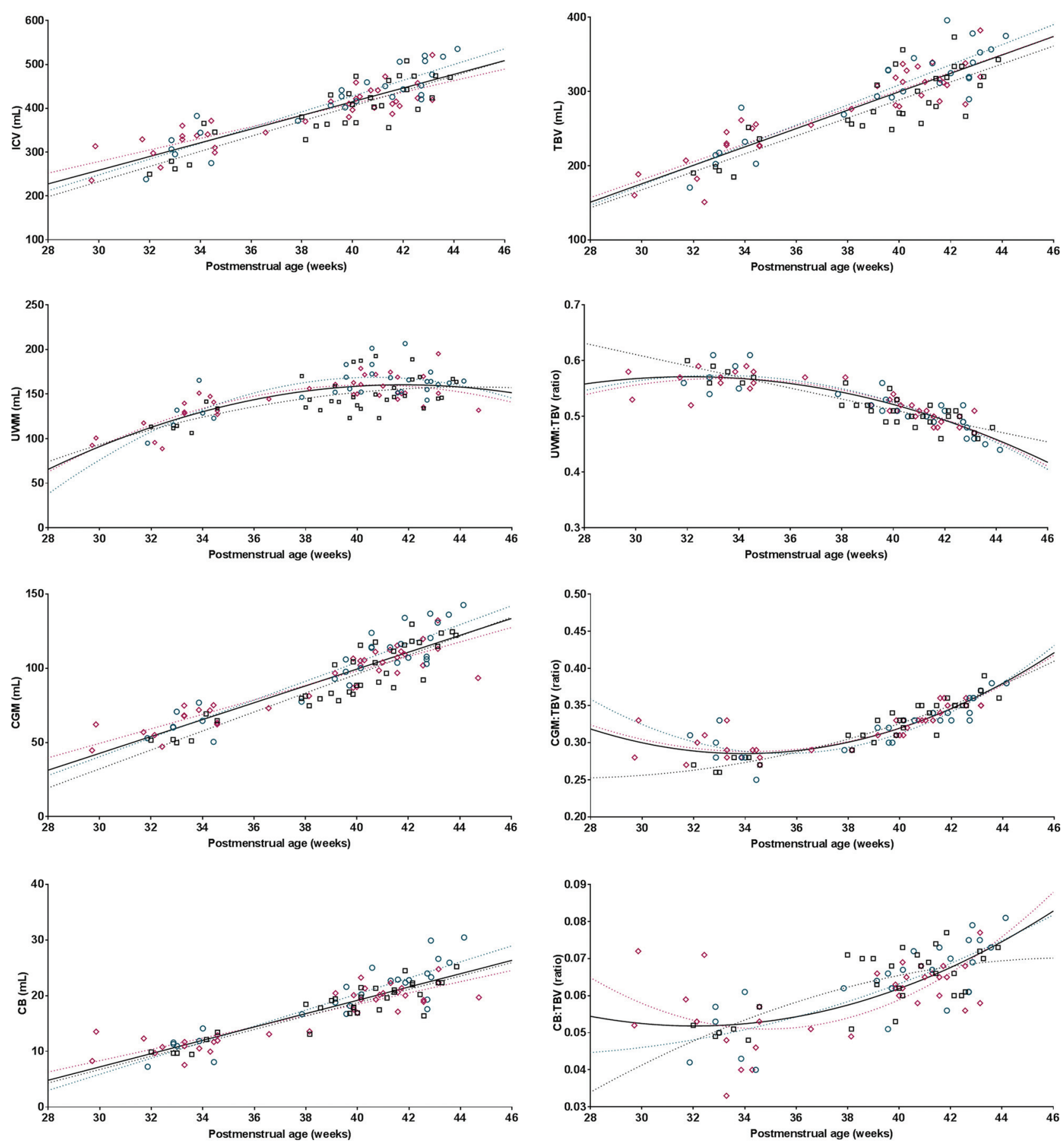

FIG 2. Fetal and neonatal brain volumes in relation to postmenstrual age. Plotted are brain volumes (y-axis) in relation to postmenstrual age at scanning ( $\mathrm{x}$-axis) for transposition of the great arteries (pink diamonds), left ventricle outflow tract obstruction (green circles), and singleventricle physiology (black squares). Either the regression or quadratic line was fitted for each congenital heart defect separately (dotted lines), also as 1 line combining all subjects (solid black line). $R^{2}$ values reflecting the goodness of fit: ICV, 0.76; TBV, 0.76; UWM, 0.53; CGM, 0.81; cerebellum, 0.79; CGM:TBV, 0.78; UWM:TBV, 0.77; cerebellum:TBV, 0.48.

portion of TBV occupied by UWM decreased with time, with a parallel increase of occupation of the TBV by CGM and the cerebellum. The proportions of VCSF: ICV and ECSF:ICV decreased during the fetal period with increasing PMA; however, it increased again in the neonatal period (Fig 3).

By means of mixed-model analysis, no differences in brain or CSF volumes were seen among CHD groups (all $F$-test $P$ values $>$ .007, with correction for multiple comparisons).

\section{DISCUSSION}

Previous studies have reported smaller brain volumes in fetuses and neonates with critical CHD compared with healthy subjects. Linking longitudinal fetal and neonatal brain and CSF volumes, for the first time, this study reveals the strong relationship between brain volumes and postmenstrual age in individuals with critical CHD. Additionally, the results show a negative correlation between fetal brain volumes and neonatal ischemic brain injury. 

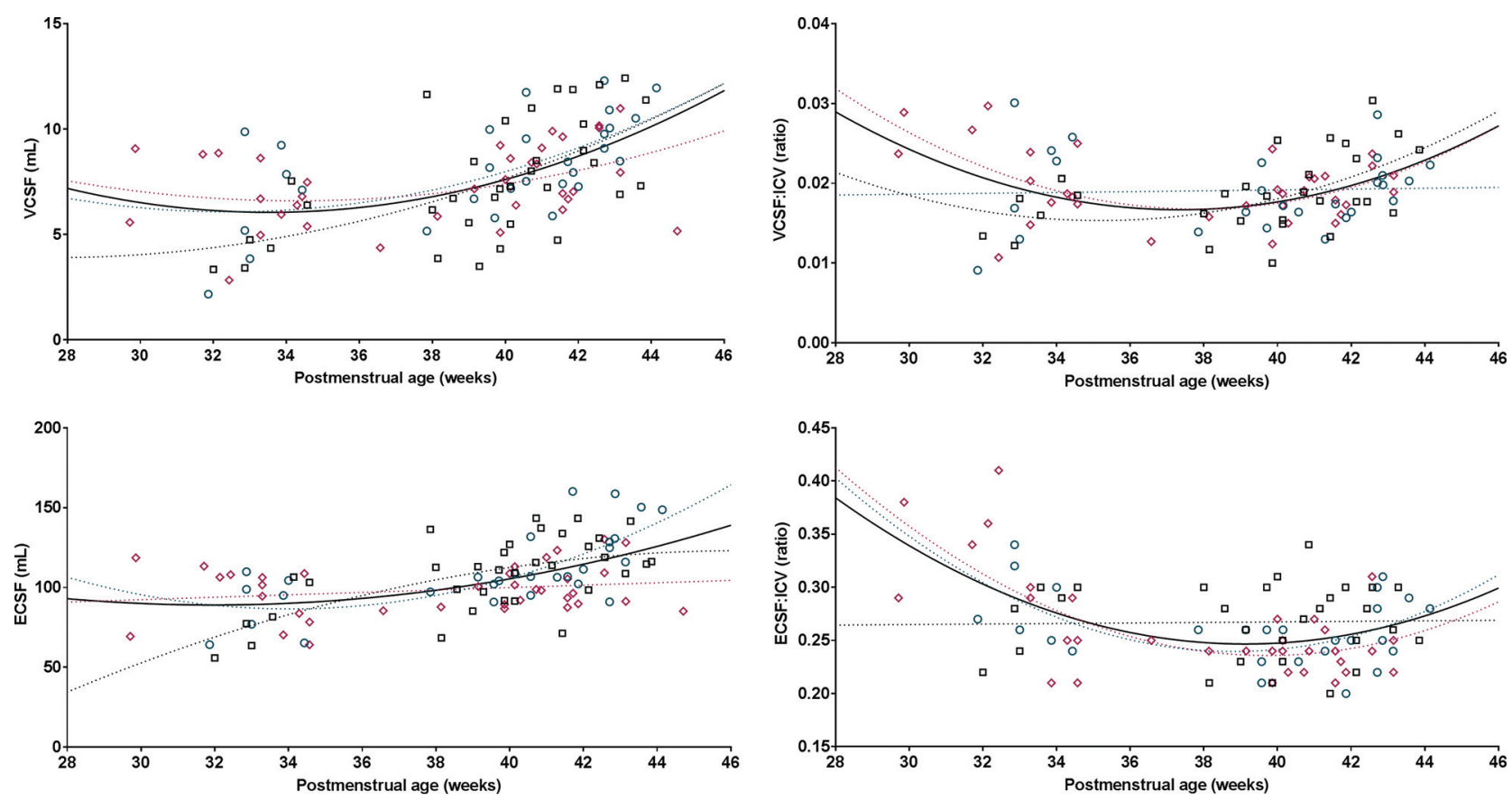

FIG 3. Fetal and neonatal CSF volumes in relation to postmenstrual age. Plotted are CSF volumes (y-axis) in relation to postmenstrual age at scanning (x-axis) for transposition of the great arteries (pink diamonds), left ventricle outflow tract obstruction (green circles), and singleventricle physiology (black squares). Either the regression or quadratic line was fitted separately for each congenital heart defect (dotted lines), also as 1 line for all subjects (solid black line). $R^{2}$ values reflecting the goodness of fit: VCSF, 0.27; ECSF, 0.27; VCSF:ICV, $0.14 ;$ ECSF:ICV, 0.24 .

Revealing the association between fetal brain imaging parameters and neonatal brain abnormalities provides important information for the potential use of fetal brain imaging parameters to estimate neonatal neurologic findings in critical CHD.

In neonates with critical CHD, volumes of the largest brain structures occupying most of the total brain volume, ie, UWM, CGM, and cerebellum, correlated with the volumes measured in the same individuals around 33 weeks of gestation. The findings of this study support the hypothesis that neonatal brain volumes are the result of antenatal brain growth and strengthen the possibility of detecting delayed brain growth at an early stage, even before birth. In our study, the association of fetal UWM and CGM was stronger with preoperative neonatal UWM and CGM than with postoperative UWM and CGM. This finding suggests that growth of UWM and CGM after birth is dependent on other neonatal factors than antenatal brain growth alone.

In our study, the association between fetal and neonatal age was less strong for the cerebellum than it was for UWM and CGM. However, the proportion of fetal TBV occupied by the cerebellum was strongly associated with the proportion of preoperative and postoperative neonatal TBV occupied by the cerebellum. The cerebellum is the brain structure with the highest increase in volume between 30 and 40 weeks PMA in extremely preterm infants. ${ }^{12}$ Previous studies have shown that in the third trimester of gestational age, cerebellar volume was comparable between fetuses with and without $\mathrm{CHD},{ }^{13,14}$ whereas at term age, a reduction of $20 \%$ in the cerebellum has been observed in neonates with critical CHD. $^{4}$

Together with the relative reduction in brain volumes with increasing gestation, a relative increase in CSF spaces has been described in fetuses with critical CHD compared with healthy fetuses. ${ }^{6}$ Larger CSF spaces are seen as an expression of brain underdevelopment. ${ }^{15}$ In healthy fetuses, CSF volumes plateau late in the third trimester, ${ }^{16}$ and the proportion of ICV occupied by CSF decreases during the third trimester from 0.4 to $0.1 .^{6}$ In our study, the proportion of ICV occupied by VCSF and ECSF was higher than 0.1 throughout the antenatal and neonatal period for all neonates with critical CHD. Absolute and proportional CSF volumes did decrease during the third trimester of gestation, to increase again in the neonatal period. In addition, the findings of our study revealed that increased absolute and proportional CSF volumes at fetal age did correlate with increased neonatal CSF volumes. This increase in CSF spaces seems abnormal and might be a sign of further brain underdevelopment or the result of brain tissue loss. Studies in older children with single-ventricle physiology have shown an association between larger CSF spaces and poorer neurodevelopment. ${ }^{17}$

Although it is becoming well-established that WM, CGM, and cerebellar volumes are smaller in fetuses and neonates with various types of critical CHD compared with healthy controls, ${ }^{4,6,13,14,18,19}$ the contribution of these findings to neurodevelopment later in childhood remains largely unknown. Smaller neonatal brain volumes are associated with abnormal neonatal neurobehavior ${ }^{20}$ and lower 6-year intelligence in children with critical CHD. ${ }^{3}$ In adolescents with critical CHD, decreased TBV is strongly correlated with poorer cognitive and executive functioning. ${ }^{21}$

In our study, ischemic and hemorrhagic brain injury was not seen in any of the fetuses. This finding supports the common thought that acquired brain injury has its onset after birth, in the neonatal intensive care and surgical period. The rate of preoperative and new postoperative neonatal ischemic injury in our pop- 
ulation is comparable with that in previous reports. ${ }^{1}$ All individuals included in this study had antenatal CHD diagnosis, which has been suggested as a protective factor for preoperative neonatal ischemic brain injury. ${ }^{2}$ In neonates with critical CHD, WMI and focal infarction are the most common forms of acquired brain injury, and especially WMI has been shown to be associated with poorer motor and cognitive outcomes in the long term. . $^{2,3}$

The results of this study revealed a correlation of smaller fetal TBV, UWM, CGM, and cerebellum with acquired neonatal ischemic brain injury (ie, moderate-severe WMI or focal infarction) in neonates with an antenatal diagnosis of critical CHD. Absolute fetal volumes of UWM, CGM, and the cerebellum (corrected for PMA) showed a stronger correlation with acquired neonatal brain injury than the proportion of TBV occupied by any of these brain structures. Previous studies have also suggested a link between neonatal brain underdevelopment and acquired neonatal brain injury. ${ }^{22,23}$ However, our study cannot reveal whether there is a causal relationship between smaller brain volumes and acquired brain injury. Brain underdevelopment might be accompanied by an increased vulnerability of the brain to disturbances in cerebral blood flow and cerebral oxygen delivery during intensive care admission and cardiac surgery.

All critical CHD groups have reduced oxygen delivery compared with healthy controls, and this reduction in brain oxygen delivery is directly associated with smaller fetal and neonatal brain volumes. ${ }^{5,7}$ Oxygen delivery is dependent on cerebral blood flow and blood oxygen content. As a result of the cardiac defect, cerebral blood flow is most severely altered in single-ventricle physiology and left-ventricle outflow tract obstruction, whereas cerebral blood oxygen content is lowest in transposition of the great arteries. ${ }^{24}$ Despite these differences in antenatal cerebral circulatory disturbances, we found no differences in growth trajectories of brain volumes among these CHD groups, reflecting most critical cardiac defects. Smaller fetal $\mathrm{TBV}^{6}$ and lower neonatal brain growth rate ${ }^{25}$ have been described in single-ventricle physiology compared with other cardiac defects; however, most neonatal studies have shown comparable reductions in the volumes of UWM and CGM among transposition of the great arteries and single-ventricle physiology. ${ }^{4,18}$ All individuals with critical CHD are at risk of reduced antenatal brain growth; however, other patient-related factors than the cardiac defect itself might determine the degree of brain underdevelopment.

This study is one of the first to explore the correlation between fetal and neonatal brain volumes using quantitative longitudinal MR imaging. This study provides important information for future research investigating whether there is a causal relationship between brain underdevelopment and brain injury, fetal brain underdevelopment as an early biomarker for neurodevelopmental outcomes, and potential strategies to improve antenatal brain growth.

This study has several limitations. First, postnatal CHD diagnosis increases the risk of preoperatively acquired ischemic brain injury, especially when low cardiac output syndrome is present. However, this population cannot be included in fetal MR imaging studies and was therefore not examined in this study. Second, absolute brain and CSF volumes are dependent on the MR imaging protocol and postimaging processing methods and are there- fore difficult to compare among studies. Third, the subgroup sample size (by heterogeneity of CHD populations) limited the possibility of performing subgroup analysis. The subgroup analysis of fetal-neonatal brain volumes by PMA is at risk of type 2 errors, potentially not showing differences among the groups.

\section{CONCLUSIONS}

Linking longitudinal fetal and neonatal brain and CSF volumes, this study reveals the strong relationship between brain volumes over postmenstrual age in individuals with critical CHD. Additionally, the results show a negative correlation between fetal brain volumes and neonatal ischemic brain injury. Combining these findings, this study suggests that fetal MR imaging can be used as an early biomarker to estimate neonatal neurologic findings in critical CHD.

\section{ACKNOWLEDGMENTS}

The authors would like to thank Gabrielle van Iperen, Raymond Stegeman, and Felix Haas for their contributions to the brain imaging program, and the Congenital Heart Disease Life Span study group of the Wilhelmina Children's Hospital in Utrecht, which includes the Department of Neonatology, Department of Pediatric Intensive Care, Department of Obstetrics, Department of Pediatric Cardiology, Department of Pediatric Cardiothoracic Surgery, Department of Pediatric Anesthesiology, Department of Radiology, Department of Medical Psychology and Child Development, and Exercise Center.

Disclosures: Ivana Isgum—UNRELATED: Grants/Grants Pending: research grant funded by Nutricia Research, University Medical Center Utrecht, Utrecht University*; Patents (Planned, Pending or Issued): I am an inventor of 1 accepted and 1 pending patent; Stock/Stock Options: I am a scientific founder and shareholder of Quantib-U B.V.; Linda S. de Vries-UNRELATED: Employment: Consultant Neonatologist at UMCU*; Grants/Grants Pending: ZonMw grant for neonatal stroke*; Payment for Lectures Including Service on Speakers Bureaus: yearly ultrasound course in London, Imperial College, sometimes honorarium for giving a lecture*; Royalties: 3 books: Hellström-Westas L, de Vries LS, Rosen I. An Atlas of Amplitude-Integrated EEGs in The Newborn. 2nd ed, Informa Health, London, August 2008; Govaert P, de Vries LS. An Atlas of Neonatal Brain Sonography, 2nd ed (CDM 182-183), Mac Keith Press, ISBN: 978-1-898683-56-8, July 2010; Volpe's Textbook Neurology of the Newborn, Elsevier, 2017.* *Money paid to the Institution.

\section{REFERENCES}

1. Claessens NH, Kelly CJ, Counsell SJ, et al. Neuroimaging, cardiovascular physiology, and functional outcomes in infants with congenital heart disease. Dev Med Child Neurol 2017;59:894-902 CrossRef Medline

2. Peyvandi S, Chau V, Guo T, et al. Neonatal brain injury and timing of neurodevelopmental assessment in patients with congenital heart disease. J Am Coll Cardiol 2018;71:1986-96 CrossRef Medline

3. Claessens NH, Algra SO, Ouwehand TL, et al; CHD Lifespan Study Group Utrecht. Perioperative neonatal brain injury is associated with worse school-age neurodevelopment in children with critical congenital heart disease. Dev Med Child Neurol 2018;60:1052-58 CrossRef Medline

4. von Rhein M, Buchmann A, Hagmann C, et al; Heart and Brain Research Group. Severe congenital heart defects are associated with global reduction of neonatal brain volumes. J Pediatr 2015;167: 1259-63 e1 CrossRef Medline

5. Kelly CJ, Makropoulos A, Cordero-Grande L, et al. Impaired development of the cerebral cortex in infants with congenital heart disease is correlated to reduced cerebral oxygen delivery. Sci Rep 2017; 7:15088 CrossRef Medline

6. Limperopoulos C, Tworetzky W, McElhinney DB, et al. Brain vol- 
ume and metabolism in fetuses with congenital heart disease: evaluation with quantitative magnetic resonance imaging and spectroscopy. Circulation 2010;121:26-33 CrossRef Medline

7. Sun L, Macgowan CK, Sled JG, et al. Reduced fetal cerebral oxygen consumption is associated with smaller brain size in fetuses with congenital heart disease. Circulation 2015;131:1313-23 CrossRef Medline

8. Lim JM, Kingdom T, Saini B, et al. Cerebral oxygen delivery is reduced in newborns with congenital heart disease. J Thorac Cardiovasc Surg 2016;152:1095-103 CrossRef Medline

9. Garel C, Alberti C. Coronal measurement of the fetal lateral ventricles: comparison between ultrasonography and magnetic resonance imaging. Ultrasound Obstet Gynecol 2006;27:23-27 Medline

10. Papile LA, Burstein J, Burstein R, et al. Incidence and evolution of subependymal and intraventricular hemorrhage: a study of infants with birth weights less than 1,500 gm. J Pediatr 1978;92:529-34 CrossRef Medline

11. Moeskops P, Viergever MA, Mendrik AM, et al. Automatic segmentation of MR brain images with a convolutional neural network. IEEE Trans Med Imaging 2016;35:1252-61 CrossRef Medline

12. Kersbergen KJ, Makropoulos A, Aljabar P, et al. Longitudinal regional brain development and clinical risk factors in extremely preterm infants. J Pediatr 2016;178:93-100.e6 CrossRef Medline

13. Schellen C, Ernst S, Gruber GM, et al. Fetal MRI detects early alterations of brain development in tetralogy of Fallot. Am J Obstet Gynecol 2015;213:392 e1-7 CrossRef Medline

14. Andescavage N, Yarish A, Donofrio M, et al. 3-D volumetric MRI evaluation of the placenta in fetuses with complex congenital heart disease. Placenta 2015;36:1024-30 CrossRef Medline

15. Brossard-Racine M, du Plessis AJ, Vezina G, et al. Prevalence and spectrum of in utero structural brain abnormalities in fetuses with complex congenital heart disease. AJNR Am J Neuroradiol 2014;35: 1593-99 CrossRef Medline
16. Andescavage NN, DuPlessis A, McCarter R, et al. Cerebrospinal fluid and parenchymal brain development and growth in the healthy fetus. Dev Neurosci 2016;38:420-29 CrossRef Medline

17. Heye KN, Knirsch W, Latal B, et al. Reduction of brain volumes after neonatal cardiopulmonary bypass surgery in single-ventricle congenital heart disease before fontan completion. Pediatr Res 2018;83: 63-70 CrossRef Medline

18. Claessens NH, Moeskops P, Buchmann A, et al. Delayed cortical gray matter development in neonates with severe congenital heart disease. Pediatr Res 2016;80:668-74 CrossRef Medline

19. Clouchoux C, du Plessis AJ, Bouyssi-Kobar M, et al. Delayed cortical development in fetuses with complex congenital heart disease. Cereb Cortex 2013;23:2932-43 CrossRef Medline

20. Owen M, Shevell M, Donofrio M, et al. Brain volume and neurobehavior in newborns with complex congenital heart defects. J Pediatr 2014;164:1121-27 el Medline

21. von Rhein M, Buchmann A, Hagmann C, et al. Brain volumes predict neurodevelopment in adolescents after surgery for congenital heart disease. Brain 2014;137:268-76 CrossRef Medline

22. Dimitropoulos A, McQuillen PS, Sethi V, et al. Brain injury and development in newborns with critical congenital heart disease. $\mathrm{Neu}$ rology 2013;81:241-48 CrossRef Medline

23. Andropoulos DB, Hunter JV, Nelson DP, et al. Brain immaturity is associated with brain injury before and after neonatal cardiac surgery with high-flow bypass and cerebral oxygenation monitoring. J Thorac Cardiovasc Surg 2010;139:543-56 CrossRef Medline

24. Donofrio MT, Bremer YA, Schieken RM, et al. Autoregulation of cerebral blood flow in fetuses with congenital heart disease: the brain sparing effect. Pediatr Cardiol 2003;24:436-43 CrossRef Medline

25. Peyvandi S, Kim H, Lau J, et al. The association between cardiac physiology, acquired brain injury, and postnatal brain growth in critical congenital heart disease. J Thorac Cardiovasc Surg 2018;155: 291-300 e3 CrossRef 